\title{
Dynamic Characteristics of Fan Impeller Combines C Ross-flow
}

\author{
Wenmin Liu \\ College of Water Conservancy \\ Yunnan Agricultural University \\ Kunming, China \\ e-mail:liuwenmin365@126.com
}

\author{
Housen Yang \\ College of Mechanical and Electrical Engineering \\ Yunnan Agricultural University \\ Kunming, China \\ e-mail:liuwenmin365@126.com
}

\author{
Xinwen Luo \\ College of Mechanical and Electrical Engineering \\ Yunnan Agricultural University \\ Kunming, China \\ e-mail:liuwenmin365@126.com
}

\author{
Xingmei Dai \\ College of Water Conservancy \\ Yunnan Agricultural University \\ Kunming, China \\ e-mail:liuwenmin365@126.com
}

\begin{abstract}
Cross-flow fan with its large volume, the export flow distribution uniformity and other advantages, has been widely used in joint harvesting. In this paper, a type of grain combines cross-flow fan be analyzed, using ANSYS Workbech finite element analysis platform, and the flow field of the cross flow fan is simulated, the dynamic characteristics of the impeller in centrifugal force, gravity and aerodynamic force are analyzed. The result is that the maximum stress of the impeller is $40.686 \mathrm{MPa}$, far less than the yield strength of the material 420MPa, the interference frequency of the impeller is $16.1 \mathrm{~Hz}$, which is far less than the vibration frequency of the first six modes of the impeller. This analysis verified the security of the wind turbine, and provided the data support for the optimization design of the wind turbine.
\end{abstract}

Keywords-Cross-flow Fan;Impeller; Fluid-solid Coupling; Flow Field; Dynamic Characteristic

\section{INTRODUCTION}

Cross-flow fan with a large amount of wind, small size, compact structure, the axial outlet flow distribution and other characteristics, widely used in agriculture cleaning equipment. Cleaning Device with combine harvester to large-scale, high-efficiency direction, original with centrifugal fans and axial fans as the combine harvester main cleaning component increasingly cannot meet the needs of production, cleaning device width increases, centrifugal fans and axial fans showed power consumption, gas distribution is uneven, large institutional size and other issues. In theory, affect the performance of the cross flow fan is not affected by the width of the cross flow fan, so the application is more and more popular in the combined harvester[1].

This will be a new type of combined harvester of cross flow fan is analyzed, the centrifugal force, gravity, aerodynamic effect, analysis of the dynamic characteristics of combined harvester of cross flow fan impeller using ANSYS Workbench.

\section{FAN MODEL}

A. The working principle of cross-flow fan

Cross-flow fan mainly consist of shell, tongue plate, impeller and other components, the shell surrounds a portion of the impeller, it is not surrounded by a large part of the impeller blades called into the gate, which is the location of the fan inlet; Another part is not enclosed fan outlet, part of the impeller this position is called a wind vane[2] .As shown in Figure 1 the structure of fan.

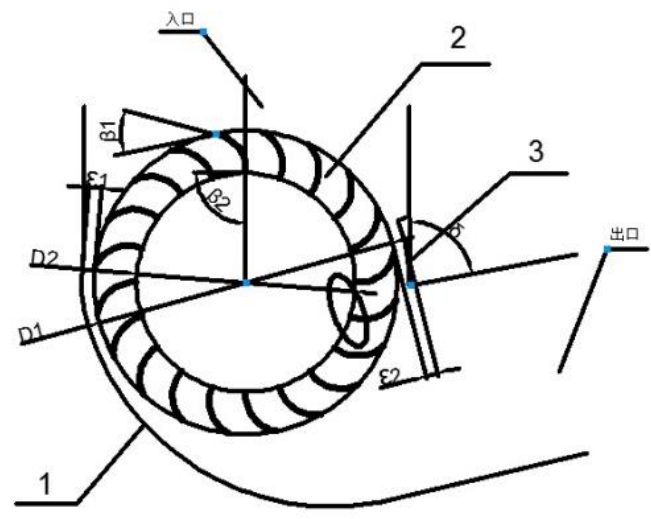

1-shell,2-impeller ,3-The tongue plate

Figure 1. Cross-flow Fan Structure Diagram

Cross-flow fan at work, the impeller rotating within the housing will be in a position near the tongue to form a vortex eccentric, eccentric vortex forming a low pressure center, a central role in this low pressure gas through the fan inlet into the interior of the fan, and then outflow fan. Theoretically, the gas flow direction in this process and the impeller shaft remain vertical, it works with centrifugal fans and axial fans are completely different[1]. 


\section{B. Fan Model}

This paper analyzes the object is a type of cross flow fan combined harvester cleaning department, and the main parameters are shown in Table 1:

TABLE I. THE MAIN PARAMETERS OF THE CROSS FLOW FAN

\begin{tabular}{|l|l|}
\hline The total width of the fan $(\mathrm{mm})$ & 1080 \\
\hline The outer diameter of the impellerD1 $(\mathrm{mm})$ & 220 \\
\hline The inner diameter of the impellerD2 $(\mathrm{mm})$ & 171 \\
\hline Impeller hub thickness $(\mathrm{mm})$ & 4 \\
\hline Impeller blade thickness $(\mathrm{mm})$ & 2 \\
\hline Notching blade within $\beta_{2}\left(^{\circ}\right)$ & 90 \\
\hline Notching outer leaves $\beta_{1}\left({ }^{\circ}\right)$ & 25 \\
\hline $\begin{array}{l}\text { Impeller housing and the rear arm spacing } \varepsilon_{1} \\
\text { (mm) }\end{array}$ & 17 \\
\hline $\begin{array}{l}\text { Impeller and guide plate spacing tongue } \varepsilon_{2} \\
\text { (mm) }\end{array}$ & 8 \\
\hline Impeller speed $(\mathrm{n} / \mathrm{min})$ & 964.9 \\
\hline
\end{tabular}

The analysis process mainly involves the fan flow field simulation analysis (CFX), static analysis and modal analysis (Static Structural) (Model). The three analyses need fan channel model and the fan impeller model. The use of parametric modeling software Creo establish crossflow fan impeller flow model and channel model, in order to analyze the results more accurate, the fan flow path is divided into the inner flow channel and outflow tract. ANSYS Workbench has a seamless interface with a variety of software, but in order to avoid problems associated with software version cannot be successfully imported model,after the modeling is complete, transforming it into IGS format. Setup complete model shown in Figure 2 and Figure 3:

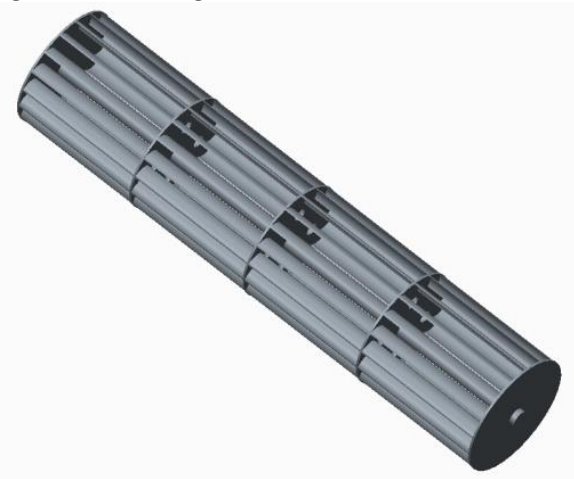

Figure 2. Fan impeller

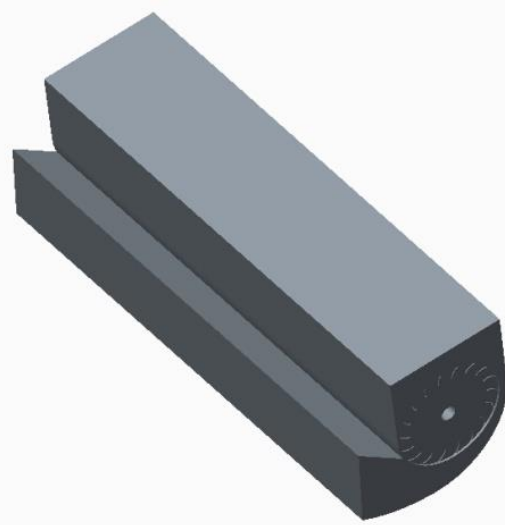

Figure 3. Fans inside and outside flow channel

\section{QUASI FAN FLOW FIELD ANALYSIS}

\section{A. Meshing}

Object Flow Field Analysis is a cross-flow fan of flow channel, the impeller is not involved in the analysis of the flow field, in the meshing, and the impeller is suppressed. Workbench provides a variety of meshing module, where the mesh using Mesh module, its high degree of automation and easy to set[3]. Since the flow path of the blade near the structure is more complex and difficult to achieve with only a mesh, researchers use the tetrahedral and hexahedral hybrid grid composed, and limit the size of the flow path of the grid for the $6 \mathrm{~mm}$,

Net outflow tract the grid size is $8 \mathrm{~mm}$. The fan flow contains 416452 nodes, 1647708 units. As shown in Figure 4.

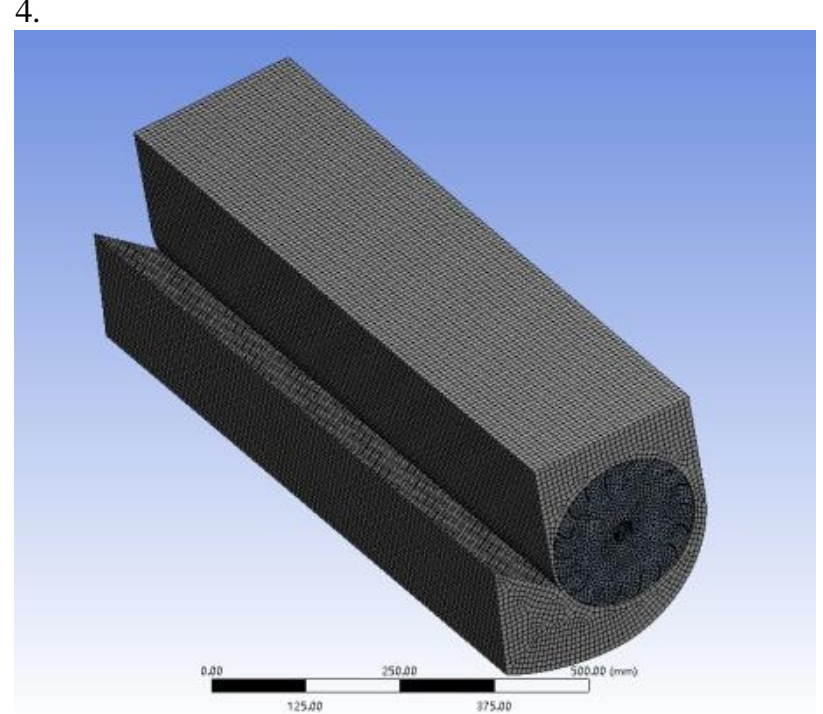

Figure 4. meshing

\section{B. Solution set}

Solving accordance with the conditions of the working conditions of the cross-flow fan is set, the type is set to solve the steady flow[3]. CFX provided material essentially covers all commonly used materials analysis, materials air at $25{ }^{\circ} \mathrm{C}$ is not considered an ideal compressibility of air and temperature dependent material, internal cross-flow fan air pressure and temperature change is not great, choose air at $25^{\circ} \mathrm{C}$ more appropriate as a fluid 
medium. Within the flow field is rotated together with the impeller, so within the flow field to set the turning axis $\mathrm{X}$ axis, speed 101rad/s. CFX provide a variety of turbulence models, turbulence model and influence the result of analysis is very large, here is a selection of K-Epsilon model that better convergence[4]. Set the reference pressure of one atmosphere.

Cross-flow fan inlet is set to a constant speed type, perpendicular to the inlet boundary inwards, is $4 \mathrm{~m} / \mathrm{s}$; Export set to free boundary, the relative pressure is zero, which does not limit the direction and velocity of the fluid at the outlet of the fan. On both sides of the channel model is set to the symmetric boundary; the other part of the shell is set to smooth fixed no slip wall condition.

There are three main solving formats of CFX: highsolving mode, upwind scheme for solving and designated mixing factor[5-6]. Where high precision high order to solve the model,this paper is to solve high-order mode; iteration number is set to 400 steps, step is set to $0.01 \mathrm{~s}$; convergence criteria set to RMS, its value is set to $1 \mathrm{e}-4$.

\section{Flow field analysis}

As shown in Figure 5, the pressure distribution on the blade of the cloud in the flow field, the pressure on the leaves of different position is not like the leaves into the wind, the wind vane and blade two position the stress difference is very big; The different side the same blade under pressure is not the same, the pressure difference size with different blade position.

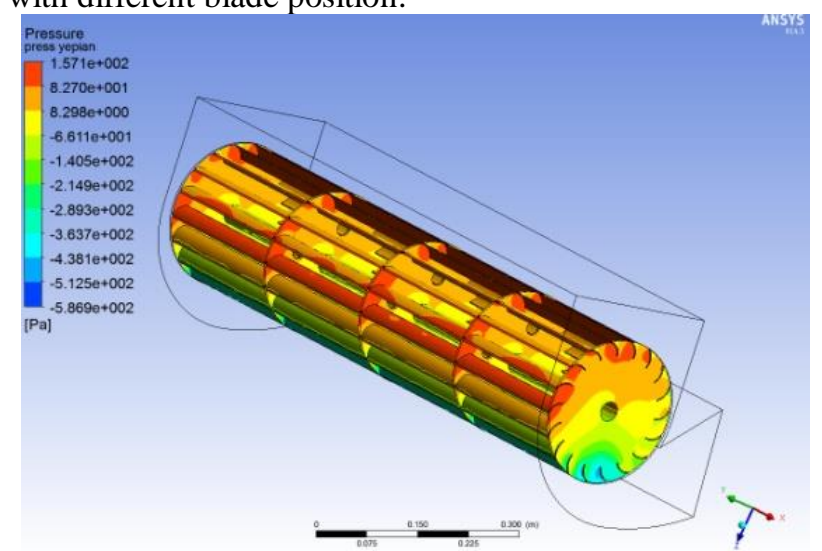

Figure 5. Nephogram of pressure distribution on the blade flow field

\section{STATIC ANALYSIS OF THE FAN IMPELLER}

\section{A. Analysis Principle}

In classical mechanics, the kinetic equation of the object as follows[7-8]:

$$
[\mathrm{M}](\mathrm{x})+[\mathrm{C}](\dot{\mathrm{x}})+[\mathrm{K}]\{\mathrm{x}\}=\{\mathrm{F}(\mathrm{t})\}
$$

Wherein: $[\mathrm{M}]$ is the mass matrix, $[\mathrm{K}]$ is the stiffness coefficient matrix, $[\mathrm{C}]$ is the damping matrix, $\{\mathrm{F}\}$ is the force loss, $\{\mathrm{x}\}$ is the displacement vector.

In the analysis of linear static force structure has nothing to do with time, so the displacement of $\{x\}$ can be obtained by the following equation:

$$
[\mathrm{K}]\{\mathrm{x}\}=\{\mathrm{F}\}
$$

The kinds of load in static analysis are: steady inertial force, displacement load, external forces and pressure, temperature, load and so on.

\section{B. Material definition and meshing}

Workbench not only provides a variety of engineering materials commonly used, but also allows users to customize the material, the material cross-flow fan impeller is QSTE420T, you need to define the material in the Workbench, and the performance is shown in Table 2:

TABLE II. QSTE420T PERFORMANCE PARAMETERS

\begin{tabular}{|l|l|}
\hline Material Name & QSTE420T \\
\hline The young's modulus $(\mathrm{GPa})$ & 200 \\
\hline Poisson's ratio & 0.3 \\
\hline Yield strength (MPa) & 420 \\
\hline Tensile strength (MPa) & 480 \\
\hline Density $\left(\mathrm{kg} / \mathrm{m}^{3}\right)$ & $7.85 \times 10^{3}$ \\
\hline
\end{tabular}

Influence of grid on the analysis results is very large, in ANSYS Workbench, the division method of the grid mainly automatic meshing, tetrahedral mesh, hexahedral dominant meshing, sweep method. This paper uses the method of automatic mesh grid, and adds some control parameters to mesh the impeller, the finite element mesh model as shown in Figure 6, a total of 431649 units and 810801 nodes.

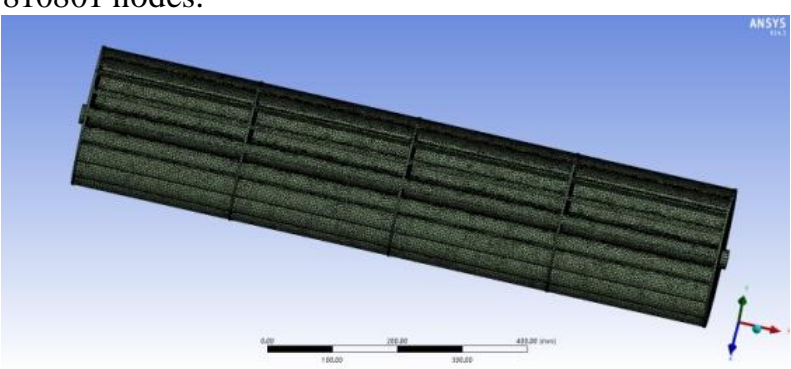

Figure 6. Impeller mesh

\section{Solving setting}

The load is considered in this paper are three: the flow field of impeller aerodynamic force, gravity, centrifugal force. Through the analysis of the flow field of CFX, this paper obtains the flow field and the force acting on the blade, Workbench can achieve data transfer between different analysis module, CFX analysis results can be imported easily to the static analysis module, which is applied on the impeller; The centrifugal force is applied through rotation speed in the impeller rotation axis axis, 101 rad/s value; gravity direction along the $\mathrm{Z}$ axis, $9.8 \mathrm{~m} / \mathrm{s} 2$ acceleration of gravity.

Cross-flow fan impeller at work, there is only one degree of freedom of rotation around the $x$-axis, where the displacement by applying a constraint on the face and side of the impeller shaft to achieve, in the $x, y, z$ direction of displacement are limited to zero constraint after only one impeller rotation around the $\mathrm{x}$-axis degree of freedom. 


\section{Solving Results}

After solving, the equivalent of the centrifugal force, gravity, a erodynamic force under the action of the impeller stress nephogram ,strain nephogram and total deformation nephogram.

As shown in Figure 7, the impeller of the equivalent stress nephogram,the maximum stress distribution in the connection hub and vane position, the maximum value is $40.686 \mathrm{MPa}$, far less than the yield strength of 420MPa. In the direction along the blade wheel, five wheel hub of the impeller is divided into four parts, the four part of the blade stress distribution is similar. Different circumferential position of the blade, the equivalent stress is not the same.

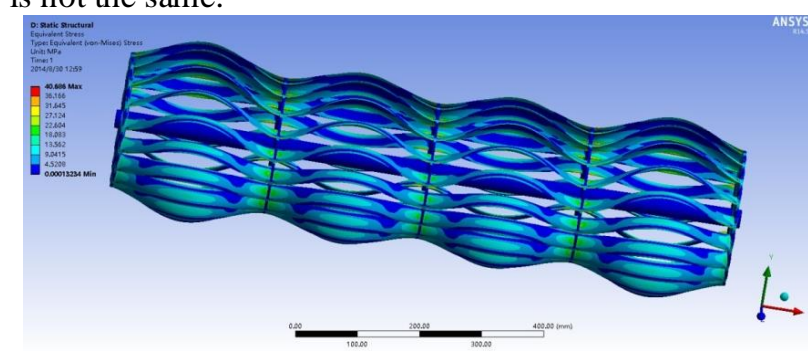

Figure 7. The impeller of the equivalent stress nephogram

As shown in Figure 8, the impeller strain nephogram is divided into four parts: the blade hub in the axial direction, each between the stress distributions is similar, where stress is large, the strain is also large. The maximum strain appears in the blades and hub connections, a maximum of $0.00020368 \mathrm{~mm} / \mathrm{mm}$. Different circumferential position of the blade, the equivalent strain is not the same.

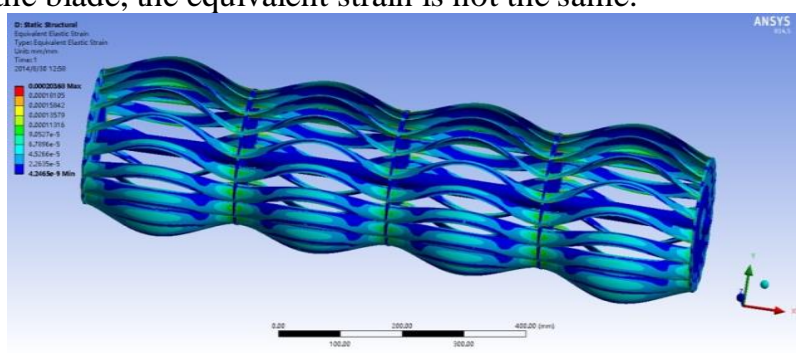

Figure 8. Impeller strain nephogram

As shown in Figure 9, the total amount of deformation of the nephogram of the impeller, the hub in the axial direction is divided into five blades; each blade of the total amount of deformation is similar to nephogram. The maximum total deformation occurs in the middle of each blade, a maximum of $0.12316 \mathrm{~mm}$. Different circumferential position of the blade, the total amount of strain is not the same.

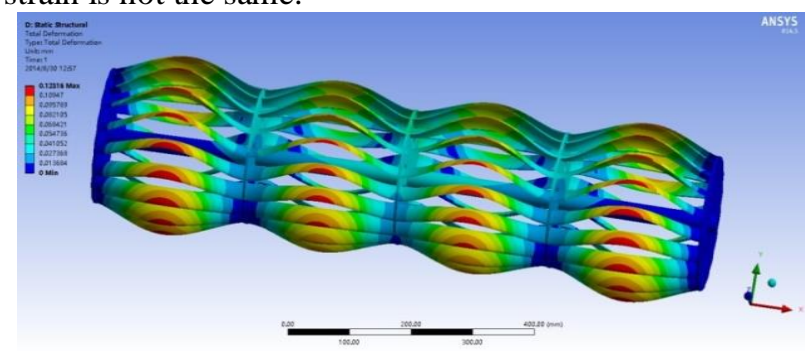

Figure 9. The total deformation nephogram of impeller

\section{PRESTRESSED MODAL ANALYSIS OF THE FAN IMPELLER}

\section{A. Analysis Principle}

Modal analysis is a numerical analysis of the vibration characteristics of the structure, is an important method to study the mechanical vibration, dynamic optimization and dynamic characteristics of structure. Modal is the inherent characteristics of the mechanical structure, modal is composed of structural geometry, material properties and constraints determined, each structure has a plurality of modes, each mode has a corresponding vibration frequency $\omega \mathrm{i}$ and vibration modes $\varnothing \mathrm{i}$, which can be calculated through the following equation:

$$
[\mathrm{K}]-\omega_{i}^{2}[\mathrm{M}]\left\{\phi_{i}\right\}=0
$$

In the formula, $[\mathrm{K}]$ is the stiffness matrix, mass matrix $[\mathrm{M}]$, and they are assumed to be constant.

Prestressed modal analysis is the vibration frequency and vibration type of prestressed concrete structure, and does not consider the prestressed modal analysis, prestressed modal analysis considering the elements and nodes of prestressed, analysis results can describe the mechanical modal characteristics under actual conditions more accurately.

This paper analyzes the centrifugal force, gravity, aerodynamic effect of the cross-flow fan impeller, its prestressed modal analysis. By the Formula 2 ([ $\sigma 0] \rightarrow$ $[\mathrm{S}]$ )and Formula 3 can be drawn prestressed modal calculation equation:

$$
[\mathrm{K}+\mathrm{S}]-\omega_{i}^{2}[\mathrm{M}]\left\{\phi_{i}\right\}=0
$$

\section{B. The analysis results}

The accuracy of the modal analysis results are influenced by the order, the greater the order, error modal analysis results will be greater. According to the needs, using the Block Lanczos method is proposed for the first six modesof cross flow fan. Vibration frequency of the first six modes is shown in Table 3.

TABLE III. THE FIRST SIX ORDER MODAL FREQUENCY

\begin{tabular}{|c|r|}
\hline Order number & Vibration frequency [Hz] \\
\hline 1 & 104.83 \\
\hline 2 & 104.84 \\
\hline 3 & 135.7 \\
\hline 4 & 265.24 \\
\hline 5 & 265.27 \\
\hline 6 & 277.34 \\
\hline
\end{tabular}

Sixth-order modal frequency in the front, appeared twice in the double root vibration equation, which is the first order and second order, fourth order and fifth order. The difference between each of the two order modal frequency is different.

Figure 10 is a first order impeller mode shapes, its vibration frequency is $104.83 \mathrm{~Hz}$, the second mode of vibration frequency is $104.84 \mathrm{~Hz}$,both vibration frequency is very close, which is double the root vibration equation, this situation often appears symmetrical structure part modal. The orders of these two modes are similar, but 
there is a phase difference of resonance. The maximum deformation occurs in the middle of the impeller. Here's nephogram is about the quality of the matrix normalized mode shapes nephogram, numerical size shown in the figure, is not part of the displacement of the real size, but the ratio of the displacement of each point[9].

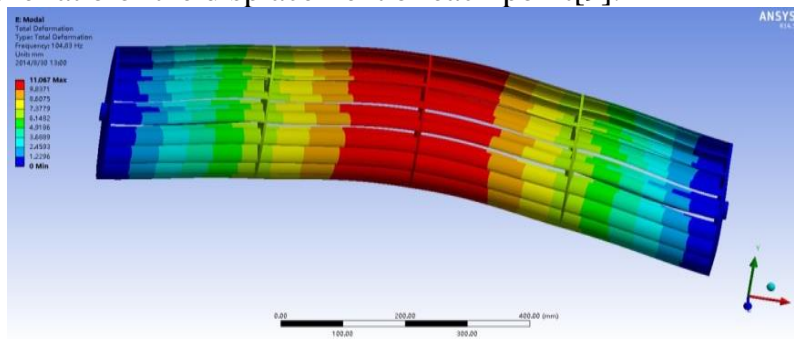

Figure 10. First order vibration type impeller nephogram

Figure 11 is a third order impeller mode shapes nephogram, the maximum deformation occurs in the middle of the impeller; and there in the center of the impeller shaft distortion.

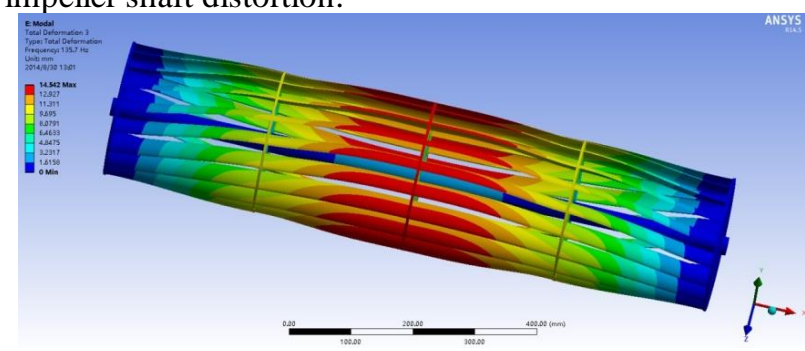

Figure 11. Third order vibration type impeller nephogram

Figure 12 is the fourth modal images of impeller, the fourth order modal frequency is similar to the fifth-order vibration frequency, the two is a double root vibration frequency vibration equation; in both vibration mode of cloud, the entire impeller into the S-type, and there is a phase difference; the maximum position is near the impeller end of the hub.

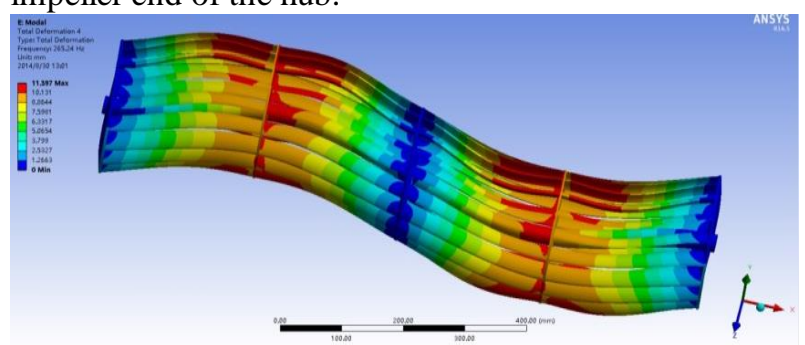

Figure 12. Fourth order vibration type impeller nephogram

Figure 13 is a sixth-order impeller mode shapes nephogram, its biggest deformation occurs in the vicinity of two wheels near the impeller section; In the blades of the two positions in the impeller shaft was greatly distorted, and two twisted in the opposite direction.

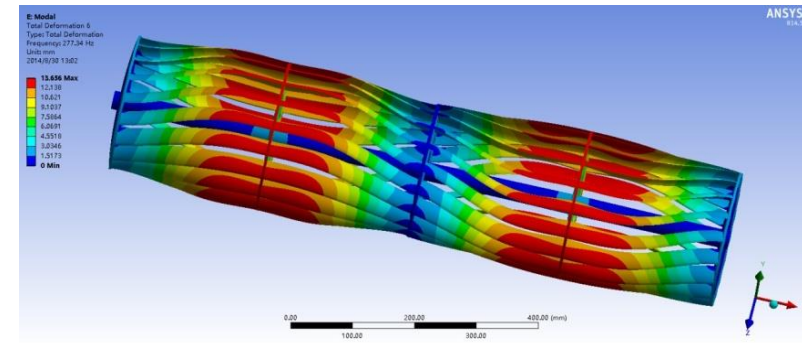

Figure 13. Sixth order vibration type impeller nephogram

The relationship between speed and frequency is[10]: $\mathrm{n}=60 \mathrm{f}$

Where, $\mathrm{f}$ is the interference frequency, the unit is $\mathrm{Hz}, \mathrm{n}$ is the rotational speed, the unit is ( $\mathrm{r} / \mathrm{min})$.

As to Equation 5 known interference frequency formula of impeller is $16.1 \mathrm{~Hz}$, which is far less than the frequency of the six modes of the impeller, when the impeller working at rated conditions, effectively avoiding the resonance hazards to ensure the safety.

\section{CONCLUSION}

1. The distribution of cross flow fan aerodynamic flow in the impeller is very complex, the maximum value is 157.1Pa, and the minimum value is $-586.9 \mathrm{~Pa}$.

2. Under the rated working condition, the maximum stress is $40.686 \mathrm{MPa}$, far less than the yield strength of $420 \mathrm{MPa}$. The impeller interference frequency is $16.1 \mathrm{~Hz}$, which is much smaller than the previous six-wheel modal vibration frequency. From the two angles of view, the impeller is absolutely safe in the working condition.

3. The maximum deformation of the impeller is $0.12316 \mathrm{~mm}$, the minimum clearance between the fan casing and the impeller is $8 \mathrm{~mm}$, from the housing and the impeller has a great influence on the fan performance, you can consider reducing the minimum clearance to increase fan efficiency.

4. Finite element analysis of ANSYS Workbench platform can improve the work efficiency, especially for the coupling analysis of multi module, multi physical field.

\section{REFERENCES}

[1] Mingjie Liu,Wei Sun,Jianguo Chang. Research on the design of [J]. agricultural mechanization for grain combine harvester, 2012 (8): 90-92.

[2] Xianjun Qiu. Application and design of cross flow fan in combine of combine harvester [J]. Journal of agricultural engineering, 2003 (1): 110-112.

[3] Bin Li,Zhengjia He,Xuefeng Chen. Workbench ANSYS design, simulation and optimization of the third edition of the Tsinghua University press, 2013:132-133.

[4] Gaowei Cong. Simulation and comparison of Dalian University of Technology [D]. numerical internal flow field for centrifugal fan, 2006.

[5] Qingshan Liu. Study on the axial force of hydraulic torque converter [D]. Changchun University of Science and Technology, 2010

[6] Fei Gao, Xin Li. CFX ANSYS 14 super learning manual [M]. Beijing: People's post press, 2013 58-91.

[7] Xinshuo Ding,Guilong Ling. ANSYS Workbench 14.5 finite element analysis case Xiangjie [M]. Tsinghua University press, 2014:87-92.

[8] Wangyu Liu,Zhangming Su. Dynamic mechanical design and research of the motor under the prestressing force of the vehicle, 2010 (6): 110-113. 
[9] Yanping Li. Finite element analysis and optimization of the centrifugal fan impeller [D]. Northwest Agriculture and Forestry University, 2012.23-24.
[10] Shaolong Wu,Yuanxiang Zhang. Yuanxiang. Based on ANSYS Workbench of high-speed electric spindle dynamics characteristic analysis [J]. Combination machine tools and automatic processing technology, 2010 (9): 20 - 26. 